\title{
Development and Validation of a Clinical Decision-Making Aid for Screening Bowel Symptoms in
}

\section{Community Pharmacies}

Deepa Sriram $\mathrm{MSc}^{1,}{ }^{*}$, Alexandra McManus PhD, MPH, PGradDipPH, BScHP(H.Biol) GAICD², Lynne Emmerton BPharm (Hons), PhD, MPS ${ }^{3}$, Richard Parsons BSc, MSc, PhD ${ }^{4}$, Moyez Jiwa MA, MD, FRCP, MRCGP, FRACGP 5

1. Study Coordinator, Department of Medical Education, Faculty of Health Sciences, Curtin University, Western Australia

2. Director, Centre of Excellence for Science Seafood \& Health (CESSH), Faculty of Health Sciences, Curtin University, Western Australia

3. Associate Professor, Pharmaceutical Practice, School of Pharmacy, Faculty of Health Sciences, Curtin University, Western Australia

4. Senior Lecturer, School of Pharmacy, Faculty of Health Sciences, Curtin University, Western Australia

5. Professor of Health Innovation, Department of Medical Education, Faculty of Health Sciences, Curtin University, Western Australia

* Corresponding Author-d.sriram@curtin.edu.au, GPO Box 1987, Perth, Western Australia 6845, 0892669581

\section{Key words}

Bowel symptoms, questionnaire, community pharmacy, advice, triage, primary health care 


\section{Abstract}

Rationale and Aim

Bowel symptoms are common, and community pharmacies are an ideal setting for health advice about these symptoms. The aim of this study was to develop and validate a questionnaire for use with adults presenting to community pharmacies with lower bowel symptoms. The purpose of the questionnaire was to stratify people into those requiring medical follow-up for symptoms, and those with self-limiting symptoms.

Method

A self-administered questionnaire, named the Jodi-Lee test (JLT), was developed in three stages: review of the literature, questionnaire design, and statistical validation against a validated screening tool, the Patient Consultation Questionnaire (PCQ) to assess the sensitivity and specificity of JLT. The questionnaire was developed to be simple, easy for all pharmacy staff to use, and require no score calculation. Its application was designed to facilitate referral from pharmacy assistants to pharmacists and from pharmacists to medical practitioners.

Results

The questionnaire comprises eight questions. It has a Flesh-Kincaid reading score of 79.5. By considering different score thresholds on the PCQ, a receiver operator characteristic (ROC) curve was calculated to assess effectiveness of the JLT. From a sample of 118 subjects, the area under the ROC curve was 0.94 . At a threshold score of 30 on the PCQ, the sensitivity was $100 \%$. The specificity was $65 \%$.

Conclusion

The JLT has high sensitivity for identifying patients with symptoms of serious bowel disease. It is also likely to identify patients who have symptoms of relatively benign disease who would benefit from medical advice. 


\section{Introduction}

Community pharmacies provide health information and services to their clients, ${ }^{1}$ and are ideal settings for health screening services. ${ }^{2,3}$ There are over 200 million occasions each year where pharmacists in Australia may be accessed for health advice and service. ${ }^{4}$ Around half of all visits to Australian community pharmacies are to buy either prescribed or over-the-counter (OTC) medication, and one-third of the visits are to ask advice about a health issue. ${ }^{5}$

Lower bowel symptoms are a common experience., ${ }^{6,7}$ A number of lower bowel diseases share clinical presentations, and certain symptom profiles are associated with serious underlying conditions such as cancer, inflammatory bowel disease or degenerative bowel conditions. ${ }^{8}$ Data suggest that one in 15 people identify the pharmacist as a source of advice about lower bowel symptoms. ${ }^{9}$

Despite this, in a survey of pharmacists in Western Australia, it was demonstrated that bowel symptoms indicative of serious disease were not recognised in a significant proportion of cases, and, more recently, that other chronic conditions were not recognised, even when clients requested over-the-counter treatments. ${ }^{10}$ Data suggest that pharmacists and general practitioners (GPs) have not consistently recognised presentations of significant colorectal pathology.$^{10-12}$ Some research supports the use of self-administered questionnaires to help identify cases that need further investigation for colorectal pathology. ${ }^{13,14}$

The CRISP (Colonoscopy Research into Symptom Prediction) Study ${ }^{14,}{ }^{15}$ developed and validated a bowel symptom questionnaire to be deployed during medical consultations. Similarly, the Patient Consultation Questionnaire (PCQ) assists doctors to prioritise colorectal referrals, and has high sensitivity for serious colorectal pathologies. ${ }^{8,16}$ The PCQ identifies people at highest risk, based on a numeric score for symptoms. ${ }^{8,13,16,17}$ It was validated in the context of patients referred to specialists by GPs. A feasibility study of the PCQ in 21 Western Australian community pharmacies identified that the majority of patients presenting to pharmacies had low PCQ scores, indicating a low risk of serious pathology. ${ }^{11}$ This is consistent with the profile of a population who seek over-the- 
counter symptomatic treatment in a developed country. ${ }^{18}$ However, there were three main problems with implementing PCQ in pharmacies:

- The PCQ has 14 questions and several sub-questions, and takes 10 minutes to complete. The consensus among pharmacy staff was that a shorter and simpler questionnaire was required.

- Reference to a computerised scoring schedule was required to rank the health of the patient from the completed PCQ, thus limiting the application of the tool in practice.

- In practice, patients may present with multiple conditions, which although identified as benign and self-limiting on the PCQ, may benefit from the advice of a GP (e.g. severe haemorrhoids, persistent diarrhoea and recurrent constipation). ${ }^{19,20}$

A simpler screening tool is needed with high sensitivity for bowel disease, to triage of clients presenting with bowel symptoms at pharmacies. In WA, the law requires that customers must interact with a pharmacy staff member before they can purchase over-the-counter products for treatment of diarrhoea and rectal bleeding. ${ }^{21}$ This affords an opportunity to test the value of a simple triage tool to help pharmacists and pharmacy staff to identify symptomatic patients who should be referred to a medical practitioner.

\section{Aim}

The aim of this study was to develop and validate a questionnaire for use with people presenting to a community pharmacy with lower bowel symptoms.

\section{Method}

A self-administered questionnaire, named the 'Jodi Lee Test' (JLT) to acknowledge the Foundation sponsoring this research (www.jodileefoundation.org.au), was developed in three stages - review of relevant literature, questionnaire construction, and statistical validation against an existing validated screening tool, the Patient Consultation Questionnaire or PCQ.

Given the limitations of the PCQ in pharmacy settings (noted in the introduction), the JLT was designed with two features in mind. Firstly, the instrument had to be usable by all pharmacy staff who interact with clients: e.g. pharmacy assistants to determine when referral to a pharmacist is 
warranted and pharmacists to determine when referral to a GP is warranted. Secondly, the instrument was intended to act as a guide to clinical decision making by the pharmacist. For these reasons, a 'check-list' approach was determined to be more appropriate in a pharmacy setting rather than to compute a risk score.

Literature Review

A systematic literature review was conducted to identify the risk factors for bowel disease. Reports of published measurement instruments were also reviewed to determine if any could be modified for our purpose. A search strategy was developed to source peer-reviewed English-language papers using CINAHL, PubMed and Scopus databases, with combinations of the following search terms: 'bowel symptoms' OR 'bowel disease'; 'questionnaire' OR 'survey' OR 'cognitive service'; 'helpseeking' OR 'general practitioner' OR 'advice'; and 'pharmacy' OR 'chemist'. The search spanned January 1990 to October 2012. The researcher and two co-investigators (DS, MJ and AM) reviewed the titles and abstracts of the articles and reached a consensus on articles that met the inclusion criteria of:

- Peer-reviewed research article (rather than letter or commentary)

- Primary focus on bowel symptoms, duration of symptoms, pharmacy as the setting for health advice, and a screening tool for bowel disease.

Additionally, the reviewers met regularly and engaged in an active dialogue about specific articles.

\section{Questionnaire Construction}

All symptoms and questions considered for inclusion in the questionnaire were reviewed by an expert panel, comprising a GP with special interest in bowel disease, a community pharmacy researcher and a public health practitioner (authors MJ, LE and AM, respectively), to enhance the face and content validity of the instrument. This was undertaken using a modified nominal group technique, whereby the researcher (author DS) generated initial questionnaire components based on discussion and the literature findings, and circulated drafts to the expert panel for several rounds of revision, with a view to retaining the minimum number of relevant items in the instrument that 
could elicit a decision about referral or not in a community pharmacy setting. Eliminating ambiguity in the wording and instructions were also considered. The nominal group technique continued until consensus was reached on all parameters noted.

Key considerations in the assessment of an at-risk client's history were identified. The questionnaire was designed for completion as a hard copy, as this was considered appropriate for use by pharmacy staff in their consultations with clients presenting with symptoms. The response options were formatted to highlight to the pharmacy staff member the client's symptoms that warranted referral. Consideration was also given to the readability of the questionnaire as the JLT will be completed by the client. Those who cannot complete the JLT themselves will be assisted by pharmacy staff. The questionnaire was checked for readability using the Flesh-Kincaid assessment system, with an aim of attaining a score between 70 and 100 (easily understood) ${ }^{2}$. The score is based on a 100-point scale: the higher the score, the easier it is to comprehend. 'Plain English' has a score of 65, which has an average sentence length of 15 to 20 words, and an average word of two syllables. ${ }^{22,23}$

\section{Statistical Validation}

The criterion validity of the JLT was assessed against the PCQ. The validation involved author DS completing a JLT for patient records from a previous study ${ }^{9,11}$ where the PCQ had been used. In cases where the researcher was unsure, two researchers assigned the JLT responses. JLT recommendations were made from 118 records. The PCQ produces an integer score between 0 and 100 for the risk of bowel disease. In the pilot study using the PCQ in community pharmacy, a score of 50 and above identified clients at higher risk of bowel cancer and in need of further investigation ${ }^{11}$. By comparison, the JLT guides the pharmacy staff member in clinical decision making about client management by highlighting a level of 'seriousness' to symptoms indicating whether further clinical investigation was recommended. The two instruments were compared to identify, using crosstabulations, the PCQ threshold score above which a participant would usually receive a referral using the JLT. The sensitivity and specificity of the JLT in identifying a case above a threshold score (ranging from 5 to 65) were calculated. Sensitivity refers to the percentage of cases with a PCQ above the 
threshold score who returned a positive JLT recommendation, while specificity indicates the percentage of those with a low PCQ (below the threshold) who returned a negative JLT recommendation. ${ }^{24}$ The Receiver Operator Characteristic (ROC) curve was obtained from these calculations to ascertain the relationship between the tests. The ROC curve displays the full picture of trade-off between the sensitivity and (1-specificity) (false positive) across a series of cut-off points. ${ }^{25}$ The area under the ROC curve is considered as an effective measure of inherent validity of a diagnostic test. This curve is useful in evaluating the discriminatory ability of this test to correctly identify subjects that require referral and those that do not; finding an optimal cut-off point so as to have the least misclassification of subjects in the referral and non-referral groups. ${ }^{3}$ The ideal characteristics of the JLT are high sensitivity (to refer clients who have a high PCQ score and therefore require follow-up) and relatively modest specificity (to not refer those who are likely not to require follow-up).

\section{Results}

Literature Review

The search produced 2,368 reports (Fig. 1). Deletion of duplicates reduced the total to 1,243. Title and abstract review further reduced the search results to 110 articles

Fig. 1 Literature Search Results

Questionnaire Construction

Of the 110 identified reports, 13 focussing on bowel symptoms were identified, and the significance, duration and associated risks of these symptoms were noted. We reviewed the following sources for potential questions that could be used or modified for our instrument the PCQ, as adapted by Ballal et $a l^{8}{ }^{8}$, the Bowel Symptom Questionnaire, as developed and validated by Adelstein et al. ${ }^{14}$, and a Web-based questionnaire-QCancer ${ }^{\circledR}$ (colorectal) for risk calculation, as adapted by Hippisley-Cox. ${ }^{26}$ Review of the literature suggested that the following symptoms indicate significant risks for chronic bowel disease: rectal bleeding; change in bowel habit; increased frequency in bowel motions; abdominal pain; abdominal discomfort; and incomplete defecation. ${ }^{13,20,27,28}$ The duration and 
frequency of the symptom(s), pain, loss of weight, anaemia and history of gastrointestinal disease were also considered key factors for diagnosis of bowel disease. ${ }^{14,26,28}$

Clarity, comprehensiveness and representativeness of each item were improved after several rounds of consultation between the expert panel. The panel identified the questions (relating to high-risk symptoms) that would warrant referral to a GP, and an appropriate order for the questions. Changes to the draft version included removal of indicators unable to be determined in a pharmacy, like 'anaemia'. Incorporation of a separate risk factor of 'discomfort in the back passage' was included as a generic easily understood measure of discomfort. The final version (Fig. 2) comprised eight questions, took approximately three minutes to complete, and mostly required tick-box responses.

Fig. 2 The Jodi Lee Test (JLT)

Content validity was determined to be satisfactory following this process. Readability of the test questions, measured by Flesch-Kincaid Grade Level, was 4, and the Flesh-Kincaid reading ease assessment resulted in a score of 79.5 (reading level 9 years of age). ${ }^{22,23}$

A referral to the GP was indicated if any of the five symptoms listed on the JLT (Q1 in Fig. 2) had been present for one week or more (Q2).

Statistical Validation

For thresholds varying from 10 to 65 , the sensitivity and specificity of the JLT to predict subjects with a high PCQ score (over the threshold) were calculated. The dataset used to perform this analysis was collected in a recent feasibility study of the use of the PCQ. ${ }^{9,11}$ The questions on the JLT were able to be answered from the responses to the PCQ. Recommendations were made for 118 PCQ score sheets using the JLT.

Table 1 illustrates the sensitivity and specificity calculated at a range of threshold PCQ score values. (Table 1. Sensitivity and Specificity of the Jodi Lee Test to Predict a PCQ Score Above or Below each PCQ Threshold Score $(n=118))$.

At a threshold score of 30 , the sensitivity was $100 \%$, which means that a user of the JLT would recommend each person with a PCQ score of 30 or more to a GP for review. For this threshold, the 
specificity of $65 \%$ indicates that $35 \%$ of people with a PCQ score less than 30 would also receive a referral (according to JLT).

These figures are illustrated in the ROC curve with sensitivity on the y-axis and 1-specificity on the $x$ axis (Fig. 3).

Fig. 3 ROC Curve

Table 2 shows the 'true' positive and 'true' negative GP referral rates of the 118 subjects.

Table 2. The Relationship between the Jodi Lee Test (JLT) Recommendation (Negative and Positive), and the PCQ Threshold Score

Thirty was identified as the threshold score, as it could potentially capture other bowel disease, while a score of 50 in the PCQ trial was chosen to focus on colorectal cancer. ${ }^{29}$ Using a threshold PCQ score of 30 (in accordance with the above findings), all 38 cases with a high score would be referred, and 52 of the 80 subjects with a low score would not be referred, if using the JLT. The remaining 28 cases with a low score would also be referred to their GP, according to the JLT.

The area under the curve $(A \cup C)$ is an overall measure of the diagnostic accuracy of an instrument. ${ }^{3}$ Each black dot on the graph corresponds to an observation: 'true' positive rate (sensitivity) and 'false' positive rate (1-specificity). The AUC in this case was 0.94 .

\section{Discussion}

The JLT a self-administered questionnaire has been developed as an aid to the consultation between pharmacy staff and client. It helps to identify patients who present with conditions that would benefit from medical referral. In this paper, we have reported the development of the JLT based on published literature and expert opinion, and its validation against a published instrument, the PCQ, to provide an alternative that guides the pharmacist in making a clinical decision without the need for computation of a score, and without the limitations around quantifying and scoring the relative importance of symptoms. The literature review emphasised the importance of pharmacy as a major source of health advice and the need to develop simple, valid screening tools that could be used within the pharmacy setting as part of the continuity of care health care model. 
Valid and reliable bowel health questionnaires are used in medical practice by GPs to aid referral to specialist care. ${ }^{17,}{ }^{23}$ The Bowel Symptom Questionnaire developed during the CRISP study ${ }^{14,} 30$ and the $\mathrm{PCQ}^{13}$ are examples of tools that GPs use to identify patients with colorectal symptoms that should be referred to specialists. There is no evidence for the efficacy of a self-administered bowel assessment questionnaire developed exclusively for use in community pharmacy.

The JLT was developed for pharmacy staff to identify and triage clients at higher risk of bowel disease. The JLT takes around three minutes to be completed and was developed to be easily assimilated into everyday practice. A controlled trial in community pharmacies is underway and will be reported elsewhere.

“'Symptoms' and 'duration' were considered the most important items in making an efficient assessment if the client might require referral. Applying clinical judgement, however, the pharmacist may consider responses to any of the other six questions of greater significance in deciding if the client requires referral. Review of the literature indicates that symptoms such as rectal bleeding, change in bowel habit, increased frequency in bowel motions, abdominal pain, abdominal discomfort and incomplete defaecation have a high relative-risk ratio for Inflammatory Bowel disease (IBD), Irritable bowel syndrome (IBS) and colorectal cancer ${ }^{13,20,27,28}$. Duration of these symptoms also plays an important factor in determining the risk. ${ }^{14,}{ }^{26}$ History of gastrointestinal diseases, and loss of weight were also considered key factors for diagnosis of bowel disease ${ }^{14,26,28}$, and were referred to in Questions 5 and 7 respectively in the JLT. Brevity in the questionnaire was essential to retain the JLT as a simple guide tool for use in a busy pharmacy setting, yet report high sensitivity for bowel disease."

The JLT was designed for use by pharmacists, much as the PCQ was designed for use by GPs. The validation of the JLT against the PCQ was successful in providing results that consistently identified clients at risk that should be referred to a GP for investigation. The additional benefit of the JLT is that it is more sensitive to less severe bowel symptoms than the PCQ thus identifying clients at an earlier stages of disease or at a point when prevention initiatives could be initiated. ${ }^{29}$ Fifty-seven 
percent of symptomatic people attending a pharmacy who completed a PCQ scored in excess of 30, demonstrating early signs of colorectal disease that might benefit from GP intervention. ${ }^{19,20,29}$ The sensitivity and specificity of a test are inversely related, that is, as sensitivity increases, the specificity decreases. ${ }^{25}$ The JLT in this study had a high sensitivity of $100 \%$ and specificity of $65 \%$; the high sensitivity may help in identifying patients at high risk of disease, the relatively low (modest) specificity may also identify patients who are unlikely to have bowel disease, but nevertheless would benefit from a GP consultation when reporting non-life-threatening pathologies. The JLT is not likely to recommend consultation for patients with short-lived self-limiting conditions that are appropriately managed with over-the-counter treatments. This is demonstrated by our data which shows that $44 \%$ (52/118) of symptomatic people would not require a GP consultation as per the JLT, and could be managed within the pharmacy. The ROC curve obtained from these values gave an AUC of 0.94 . AUC measures discrimination ${ }^{3}$, that is, the ability of JLT to correctly classify those with and without referrals. The large AUC of 0.94 indicates the favourable overall performance of the Jodi Lee Test to identify patients at risk of serious bowel pathology.

The aim of the study was to identify those who have symptoms that may indicate serious or benign bowel disease that would benefit from further generalist medical assessment. We used the PCQ as a proxy independent measure of the endpoint, which is the GP consultation. While the PCQ has been validated for serious bowel disease at high cut-off score of 70 or more, a lower cut-off may indicate emerging disease or other bowel conditions that require GP intervention, and are appropriately identified in a community pharmacy setting. ${ }^{16}$

Limitations of the Study

In this study, the JLT was tested against the PCQ. In ideal research practice, patients would complete both the PCQ and the JLT, and criterion validity would be established by demonstrating similar outcomes from the application of both tests. In this study, the key questions in the JLT match some of the questions in the PCQ; therefore, we obtained approval to use an existing database of PCQ responses to generate responses to the JLT, effectively achieving the same outcome as the patient 
responding to both questionnaires. The scores of the PCQ were used to determine the sensitivity and specificity of the JLT. We acknowledge that this was a departure from usual practice and that may have perpetuated bias. The next step is a prospective evaluation of the JLT against the detection of pathology to further confirm the validity of the JLT in practice.

The other limitation of the developed questionnaire is that as many as $35 \%$ of cases identified by the JLT as warranting referral have a PCQ score of less than 30 , suggesting that these subjects are at low risk of bowel disease. The JLT has been shown to perform well (sensitivity) at a PCQ threshold score of 30 , but with modest specificity (65\%). While other decision-making tools incorporate scoring systems, ${ }^{29}$ the JLT acknowledges the importance of clinical judgement in client consultations. The pharmacist can override the referral recommendation if he/she feels that the symptoms may be due to factors other than underlying (or developing) disease. It is possible that the client may have already seen a doctor recently regarding the presenting symptoms which would be indicated in Question 6 (Q6) of JLT, or that the symptom might be a side effect of some medication that the client is taking (Q7). These items were considered a matter of clinical judgement rather than something that could be incorporated as a reason for referral. This would lead to further refinement by the pharmacists which may improve specificity. These issues can be studied in detail during a prospective study to test the use of the JLT in community pharmacies.

\section{Conclusion}

The JLT has demonstrated high sensitivity and a modest specificity for identification and triage of symptoms of bowel disease, particularly at the more serious end of the spectrum of symptoms likely to present to community pharmacies.

The utility of any new diagnostic test lies in its ability to clearly delineate cases which require medical advice. In the current drive for health promotion initiatives within community pharmacy, there is a need for the development of user-friendly, valid triage instrument that have the potential to improve pharmacy practice. A pharmacy staff-led questionnaire such as the JLT should assist the pharmacy staff to identify and triage patients at moderate to high risk of bowel disease. 


\section{Acknowledgement}

I would like to acknowledge the Jodi Lee Foundation for sponsoring this research.

(www.jodileefoundation.org.au) 


\section{References}

1. Bjorkman, I., Viberg, N., Rydberg, L., Stalsby Lundborg, C. (2008) Health promotion at Swedish pharmacies - views of the staff. Pharmacy Practice, 6,211 - 8.

2. Pande, K. C., de Takats, D., Kanis, J. A., Edwards, V., Slade, P., McCloskey, E. V. (2000) Development of a questionnaire (OPQ) to assess patient's knowledge about osteoporosis. Maturitas, $37(2), 75-81$.

3. Indrayan, A. (2012) Medical Biostatistics. In, Boca Raton, FL: Chapman \& Hall/CRC Press.

4. Anderson, N., Thornberry, F., Emerson, L. (2003) The Emergency Locum Service: meeting rural pharmacists' emergency needs. In.

5. Chapman, C., van den Bosch, D. (2010) The nature, extent and impact of triage provided by community pharmacies in Victoria. Pharmacy Guild of Australia.

6. Curless, R., French, J., Williams, G. V., James, O. F. (1994) Comparison of gastrointestinal symptoms in colorectal carcinoma patients and community controls with respect to age. Gut, 35(9),1267-70.

7. Heaton, K., O'Donnell, L., Braddon, F., Mountford, R., Hughes, A., Cripps, P. (1992) Symptoms of irritable bowel syndrome in a British urban community: consulters and nonconsulters.

Gastroenterology, 102(6),1962.

8. Ballal, M., Selvachandran, S., Maw, A. (2010) Use of a patient consultation questionnaire and weighted numerical scoring system for the prediction of colorectal cancer and other colorectal pathology in symptomatic patients: A prospective cohort validation study of a Welsh population. Colorectal Disease, 12(5),407-14.

9. Jiwa, M., Sargant, S., Hughes, J., O'Connor, M., Hodder, R. (2009) Triaging consumers who present bowel symptoms to community pharmacies: a pilot study of two interventions. Australian Pharmacist, 28,516-20.

10. Jiwa, M., Spilsbury, K., Duke, J. (2010) Do pharmacists know which patients with bowel symptoms should seek further medical advice? A survey of pharmacists practicing in community pharmacy in Western Australia. The Annals of pharmacotherapy, 44(5),910-7.

11. Jiwa, M., Sriram, D., Khadaroo, Z., Ping-Delfos, W. C. S. (2011) Could community pharmacies offer an opportunity to improve outcomes for patients with bowel cancer? Quality in Primary Care, 19(2),105-8.

12. Jiwa, M., Gordon, M., Arnet, H., Ee, H., Bulsara, M., Colwell, B. (2008) Referring patients to specialists: a structured vignette survey of Australian and British GPs. BMC Family Practice, 9(1),2.

13. Selvachandran, S., Hodder, R., Ballal, M., Jones, P., Cade, D. (2002) Prediction of colorectal cancer by a patient consultation questionnaire and scoring system: a prospective study. The Lancet, 360(9329),278-83.

14. Barbara-Ann, A., Les, I., Petra, M., Peter, K., David, J., Les, B. (2008) A self administered reliable questionnaire to assess lower bowel symptoms. BMC Gastroenterology, 8.

15. Adelstein, B.-A., Macaskill, P., Turner, R. M., Katelaris, P. H., Irwig, L. (2011) The value of age and medical history for predicting colorectal cancer and adenomas in people referred for colonoscopy. BMC Gastroenterology, 11(1),97.

16. Rai, S., Ballal, M., Thomas, W., Miller, A., Jameson, J., Steward, W. (2008) Assessment of a patient consultation questionnaire-based scoring system for stratification of outpatient risk of colorectal cancer. British Journal of Surgery, 95(3),369-74.

17. Pugh, W., Porter, A. M. (2011) How sharp can a screening tool be? A qualitative study of patients' experience of completing a bowel cancer screening questionnaire. Health Expectations, 14(2),170-7.

18. Green, L. A., Fryer Jr, G. E., Yawn, B. P., Lanier, D., Dovey, S. M. (2001) The ecology of medical care revisited. New England Journal of Medicine, 344(26),2021-5.

19. BURKE, C., Bianchi, L. K. (2005) Colorectal Neoplasia. The Year in Gastroenterology and Hepatology, 1,71. 
20. National Institute for Health and Clinical Excellence. (2005) Referral guidelines for suspected cancer (Clinical guideline 27). http://www.nice.org.uk/nicemedia/live/10968/29814/29814.pdf. In, London.

21. Therapeutic Goods Administration. ( 2011) Poisons Standard. Australian Government Department of Health and Ageing, Canberra.

22. Mcinnes, N., Haglund, B. J. A. (2011) Readability of online health information: implications for health literacy. Informatics for Health and Social Care, 36(4),173-89.

23. Flesch. RF. (1974) The art of readable writing. Harper and Row, New York.

24. Löwe, B., Gräfe, K., Zipfel, S., Witte, S., Loerch, B., Herzog, W. (2004) Diagnosing ICD-10

Depressive Episodes: Superior Criterion Validity of the Patient Health Questionnaire. Psychotherapy and Psychosomatics, 73(6),386-90.

25. Kumar, R., Indrayan, A. (2011) Receiver operating characteristic (ROC) curve for medical researchers. Indian pediatrics, 48(4),277-87.

26. Hippisley-Cox, J., Coupland, C. (2012) Identifying patients with suspected colorectal cancer in primary care: derivation and validation of an algorithm. The British Journal of General Practice, 62(594),e29.

27. Kolfenbach, L. (2007) The pathophysiology, diagnosis, and treatment of IBS. JAAPA : Journal of the American Academy of Physician Assistants, 20(1),16-20.

28. Astin, M., Griffin, T., Neal, R. D., Rose, P., Hamilton, W. (2011) The diagnostic value of symptoms for colorectal cancer in primary care: A systematic review. British Journal of General Practice, 61(586),e231-e43.

29. Smith, D., Ballal, M., Hodder, R., Soin, G., Selvachandran, S., Cade, D. (2006) Symptomatic presentation of early colorectal cancer. Annals of the Royal College of Surgeons of England, 88(2),185.

30. Barbara-Ann, A., Macaskill, P., Turner, R., Katelaris, P., Irwig, L. (2011) The value of age and medical history for predicting colorectal cancer and adenomas in people referred for colonoscopy. BMC Gastroenterology, 11(1),97. 


\section{Figure Legend}

Fig. 1 - Literature Search Results: page number 19

Fig. 2 - The Jodi Lee Test (JLT): page numbers 20,21

Fig. 3 - ROC curve: page 22

Tables

Table 1. Sensitivity and Specificity of the Jodi Lee Test to Predict a PCQ Score Above or Below each PCQ Threshold Score $(n=118)$ : page 17

Table 2. - The Relationship between the Jodi Lee Test (JLT) Recommendation (Yes or No), and the PCQ Threshold Score: page 18 
Table 1. Sensitivity and Specificity of the Jodi Lee Test to Predict a PCQ Score Above or Below each PCQ Threshold Score $(n=118)$

\begin{tabular}{|c|c|c|}
\hline $\begin{array}{r}\text { Threshold } \\
\text { score }\end{array}$ & Sensitivity & Specificity \\
\hline 5 & 68.8 & 100.0 \\
\hline 10 & 75.6 & 96.9 \\
\hline 15 & 81.9 & 84.8 \\
\hline 20 & 84.8 & 80.8 \\
\hline 25 & 88.5 & 69.7 \\
\hline 30 & 100.0 & 65.0 \\
\hline 35 & 100.0 & 61.2 \\
\hline 40 & 100.0 & 57.8 \\
\hline 45 & 100.0 & 54.7 \\
\hline 50 & 100.0 & 51.5 \\
\hline 55 & 100.0 & 49.1 \\
\hline 60 & 100.0 & 46.8 \\
\hline 65 & 100.0 & 46.0 \\
\hline
\end{tabular}


Table 2. The Relationship between the Jodi Lee Test (JLT) Recommendation (Negative and Positive), and the PCQ Threshold Score

\begin{tabular}{|l|l|l|l|l|c|}
\hline \multirow{2}{*}{ PCQ Threshold score } & \multicolumn{3}{|l|}{ JLT recommendation } & \multirow{2}{*}{ Total } \\
\cline { 2 - 5 } & \multicolumn{2}{|l|}{ Negative } & \multicolumn{2}{|l|}{ Positive } & \multirow{2}{*}{} \\
\cline { 2 - 5 } & $\mathrm{N}$ & $\%$ & $\mathrm{~N}$ & $\%$ & \\
\hline$<=30$ & 52 & 65 & 28 & 35 & 80 \\
\hline $31+$ & 0 & 0 & 38 & 100 & 38 \\
\hline Total & 52 & & 66 & 118 \\
\hline
\end{tabular}

$\mathrm{N}$ - number

52 is 'True' Negative, 0 - ‘False' Positive, 28 - 'False' Negative, 38 - 'True' Positive 
Fig. 1 Literature Search Results

\begin{tabular}{|l|l|}
\hline "bowel & "bowel \\
symptoms" OR & symptoms" OR \\
bowel AND & bowel AND \\
tools OR survey & $\begin{array}{l}\text { pharmacy OR } \\
\text { OR }\end{array}$ \\
questionnaire & OR chemist - \\
OR "cognitive & 24 \\
service" - 1,109 & \\
& \\
\end{tabular}

\begin{tabular}{|l||l|}
\hline $\begin{array}{l}\text { "bowel } \\
\text { symptoms" OR }\end{array}$ & $\begin{array}{l}\text { "bowel } \\
\text { disease" OR } \\
\text { bowel AND } \\
\text { "primary }\end{array}$ \\
"bowel \\
health care" - \\
116 & 1,119 \\
& \\
& \\
& \\
\hline
\end{tabular}

\begin{tabular}{|c|c|c|c|}
\hline \multirow[b]{2}{*}{$\begin{array}{l}\text { Screened titles } \\
\text { and abstracts } \\
\text { of } 643 \text { articles } \\
\text { that met the } \\
\text { inclusion } \\
\text { criteria }\end{array}$} & \multirow[b]{2}{*}{$\begin{array}{l}\text { Screened titles } \\
\text { and abstracts } \\
\text { of } 7 \text { articles } \\
\text { that met the } \\
\text { inclusion } \\
\text { criteria }\end{array}$} & \multicolumn{2}{|c|}{$\begin{array}{l}\text { Duplicates removed - 1,243 total } \\
\text { articles for further screening }\end{array}$} \\
\hline & & $\begin{array}{l}\text { Screened titles } \\
\text { and abstracts } \\
\text { of } 69 \text { articles } \\
\text { that met the } \\
\text { inclusion } \\
\text { criteria }\end{array}$ & $\begin{array}{l}\text { Screened titles } \\
\text { and abstracts } \\
\text { of } 524 \text { articles } \\
\text { that met the } \\
\text { inclusion } \\
\text { criteria }\end{array}$ \\
\hline$\downarrow$ & $\vee$ & $\vee$ & $\vee$ \\
\hline $\begin{array}{l}48 \text { relevant } \\
\text { articles } \\
\text { identified }\end{array}$ & $\begin{array}{l}6 \text { relevant } \\
\text { articles } \\
\text { identified }\end{array}$ & $\begin{array}{l}16 \text { relevant } \\
\text { articles } \\
\text { identified }\end{array}$ & $\begin{array}{l}40 \text { relevant } \\
\text { articles } \\
\text { identified }\end{array}$ \\
\hline
\end{tabular}

Total of 110 relevant articles 
Fig. 2 The Jodi Lee Test (JLT)

Q1. Are you experiencing any of the following symptoms?

(tick $\underline{\mathbf{A L L}}$ that apply)

Diarrhoea (loose, watery and frequent bowel motions)

Constipation

Alternating constipation and diarrhoea

Bleeding from the back passage

Discomfort at your back passage (soreness, itch, lump)

Q2. How long have you had these symptoms?

Less than 1 week

1 week or more

Q3. Is this unusual for you?

Yes

No

Q3a. If yes, in what way is it unusual?

Q4. Is this symptom(s) associated with any pain?

Yes

No

Q4a. If yes, what is the pain like? (circle the number that describes the pain)

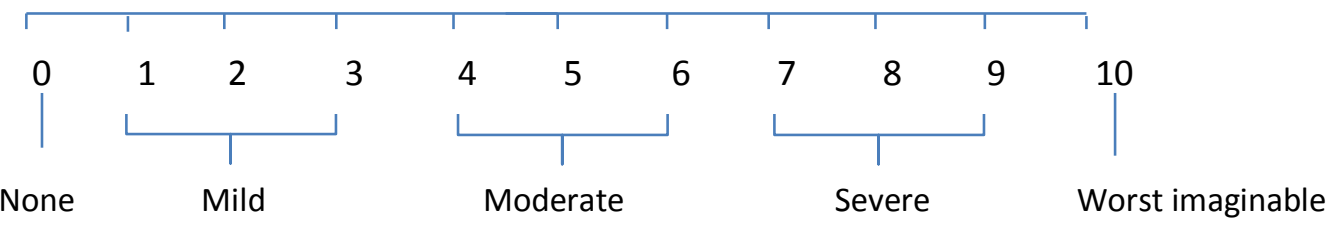

Q5. Have you lost weight unexpectedly in the past 4 weeks?

$$
\begin{aligned}
& \text { Yes } \\
& \text { No }
\end{aligned}
$$

Q5a. If yes, approximately how much weight have you lost? $\mathrm{kg}$

Q6. Have you talked to a doctor about this symptom(s)? 
Yes

No

Q6a. If yes, when was the last time you talked to the doctor about this symptom(s)?

Q7. Have you had any bowel problems in the past 12 months?

Haemorrhoids (Piles)

Colitis

Polyps

Cancer

Other

Q8. Please list any medication that you are currently taking (including medicines bought without a prescription, and natural products). 
Fig. 3 ROC Curve

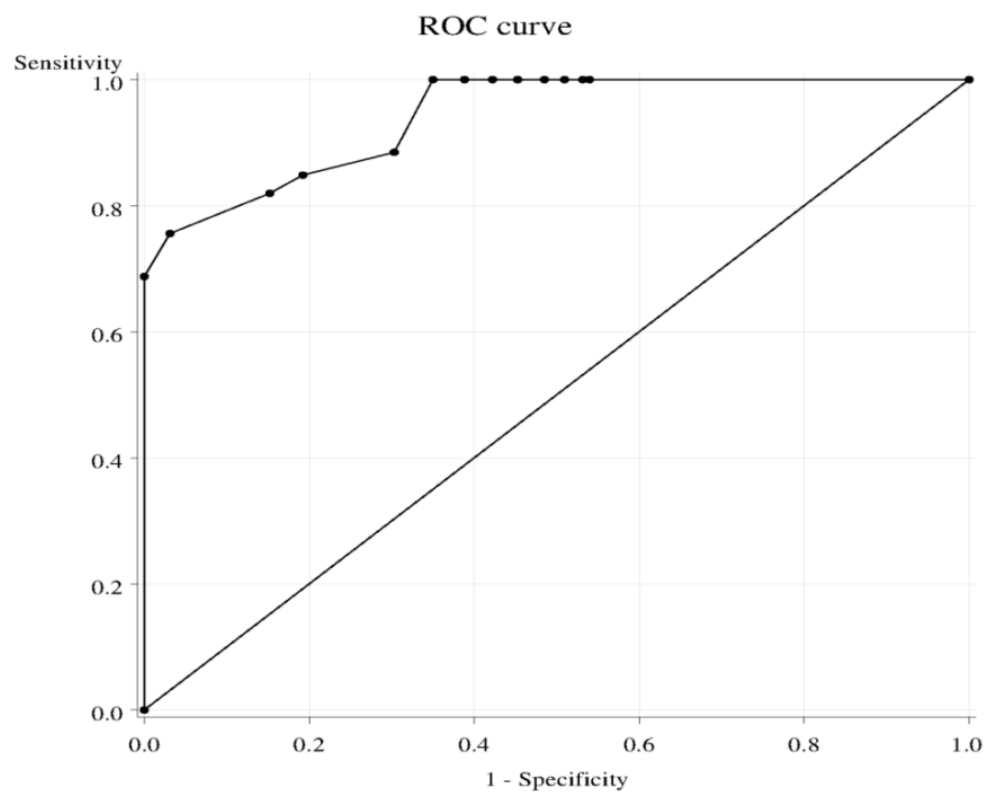

